\title{
Trends in GP prescribing of psychotropic medications among young patients aged 16-24 years: a case study analysis
}

\author{
Bianca Brijnath ${ }^{1,2^{*}} \mathbb{D}$, Ting Xia ${ }^{2}$, Lyle Turner $^{2}$ and Danielle Mazza ${ }^{2}$
}

\begin{abstract}
Background: Current clinical guidelines recommend non-pharmacological interventions as first-line treatments for young patients aged 16-24 years with a mental health condition (MHC). However, several studies have noted increasing trends in psychotropic prescribing for this age group, especially in antidepressant prescribing. In Australia, the vast majority of psychotropic medications prescribed to young people come from the general practice setting. To assess whether Australian General Practitioners (GPs) are prescribing in accordance with clinical guideline recommendations, this study examined trends in GP prescribing of psychotropic medications to young patients aged 16-24 years.
\end{abstract}

Methods: We performed a retrospective analysis of routine general practice data from 9112 patients aged 16-24 years with a MHC. Data were extracted from the Melbourne East Monash General Practice Database from 1/01/2009 to 31/12/2014. The main outcome measures included the number of consultations for patients with MHCs, psychotropic prescribing by GPs, and patient characteristics associated with the likelihood of being prescribed a psychotropic.

Results: In total, 9112 out of a total of 77,466 young patients were identified as having a MHC in this study, and 11,934 psychotropic prescriptions were provided to 3967 (43.5\%) of them over the study period. Antidepressants accounted for $81.4 \%$ of total psychotropic prescriptions, followed by anxiolytics (9.6\%) and antipsychotics (9.0\%). The number of prescriptions issued to individuals with MHCs increased over time. Women and patients aged 21-24 years had higher incidence rates for prescription than men and those aged 16-17 (IRR: 1.15, 95\% Cl 1.08-1.22, IRR: 1.93, 95\% Cl 1.750-2.11).

Conclusions: Our findings demonstrate an increasing trend in GP prescribing of psychotropics to young people over the study period with higher levels of prescribing to women and those 21-24 years of age. Although GP prescribing corresponded with guideline recommendations on the whole, there were discrepancies between GP's antidepressant prescribing and guideline recommendations, reasons for which were unclear. Research is needed to investigate GPs decision-making processes underlying their prescribing, to target interventions to improve existing data in GP records to improve management, and to identify areas of further training if needed to facilitate greater concordance between clinical practice and guideline recommendations.

Keywords: Australia, General practitioners (GPs), Youth, Mental health, Psychotropic, Prescribing

\footnotetext{
* Correspondence: bianca.brijnath@curtin.edu.au

${ }^{1}$ School of Occupational Therapy and Social Work, Faculty of Health Sciences, Curtin University, Building 401, Bentley Campus, Perth 6152, Australia

${ }^{2}$ Department of General Practice, School of Primary Care, Faculty of Medicine

Nursing and Health Sciences, Monash University, Melbourne, Australia
} 


\section{Background}

In Australia, over $89 \%$ of antidepressants and $70 \%$ of antipsychotics prescribed to young people aged 15 to 24 are prescribed by General Practitioners (GPs) [1]. UK, Canadian, and Australian studies show that GPs face many challenges in managing mental illness for this patient group, such as grappling with the unique behavioural and biological changes associated with adolescence; navigating the difficulties associated with triadic consults (i.e., where a third party such as a parent or friend might be present during the GP consult with a young person); negotiating different expectations by young people and families around socially accepted behaviours regarding alcohol and substance use, use of digital media, and relationships between teenagers and parents; working out ways to forge effective and trusting therapeutic relationships with young people; [2] and treating severe and persistent mental illness [3, 4]. Studies show that GPs are apprehensive about overmedicalising young patients [5] and are less likely to prescribe psychotropics if their young patient's hold negative views about psychotropics [6], express a preference for non-pharmacological treatments [7], or are new patients at their clinic [8]. Health warnings also reduce the likelihood of prescriptions; for example, the imposition of US and European regulator suicidality warnings on Selective Serotonin Reuptake Inhibitors (SSRIs) use in young people resulted in a decline in SSRI prescriptions among US and Dutch clinicians [9].

Current guidelines for youth mental health in Australia recommend prescribing only those psychotropics with the best evidence base and safest profile in the lowest effective dose for the shortest time possible (Table 1) [10-12]. These guidelines concord with guideline recommendations from the UK and US, where the philosophy "start low and go slow" underlies nearly all recommendations regarding psychotropic prescribing in young people $[13,14]$. Previous analysis from two

Table 1 Summary of guideline recommendations for psychotropic prescribing for youth mental health [10-12]

- Non-pharmacological interventions are the first line of treatments for $\mathrm{YMH}$

- Medication treatment should follow thorough assessment and diagnosis and be part of a comprehensive care plan

- Only those psychotropics with the best evidence base and safest profile should be prescribed

- Prescriptions should be in the lowest effective dose for the shortest time possible

- Fluoxetine, the only SSRI with a strong evidence base, should be considered following unsuccessful psychological therapy for reduction of moderate to severe depressive symptoms in adolescents

- Benzodiazepines are generally not recommended for use in children.

- Ongoing monitoring following psychotropic prescriptions is critical.
Australian national general practice clinical audits (1998-1999 and 2000-2002) showed GP psychotropic prescribing was conservative for youth mental health with non-pharmacological treatments alone, or in combination with psychotropics, being the preferred option [15]. However, this data is over 15 years old and changes in Australian primary care, such as the implementation of the Better Access to Mental Health Care Scheme (in which GPs can refer patients to mental health specialists for up to 10 free sessions per year), and availability of new psychotropics (e.g. Serotonin-norepinephrine reuptake inhibitors, or SNRIs) may have influenced GP prescribing patterns for youth mental health. Longitudinal trends in GP prescribing [16] and pharmacy dispensing [17] for psychotropic medications suggest this is the case for the general adult population. However, less is known about GP psychotropic prescribing to young people. Accordingly, in this paper, we conducted a noncomparative case study analysis to examine trends in GP psychotropic prescribing to patients aged 16-24 years over a 5 year period.

\section{Methods}

Data were drawn from the Melbourne East Monash General Practice Database (MAGNET) a collaboration between Monash University and the Melbourne East GP network. MAGNET comprises data routinely collected from the computerised medical records of patients attending 50 general practices within the inner eastern Melbourne region and is ethics approved from Monash University.

We analysed data from 1 January 2009 to 31 December 2014 for all patients aged 16-24 years at the time of consultation. Eligibility criteria for inclusion in the study were: Patients had a diagnosis of a mental health condition (MHC) in their clinical record and/or had been prescribed a psychotropic medication during the study period. Patients were identified as having a mental health diagnosis through examination of coded diagnoses attached to their computerised medical record [18].

Diagnoses were coded into high-level categories reflecting the primary problem description or diagnosis using a computerised algorithm [18]. Psychotropic medications were coded according to the Anatomical Therapeutic Chemical classification system. This system is used for the classification of active ingredients of drugs according to the organ or system on which they act and their therapeutic effect. Medication records were excluded for patients aged above 25 at the time of prescription.

For each patient we extracted demographic characteristics (age, gender, socioeconomic status, postcode, smoking, and alcohol status), consultation information (the clinic location and the consultation dates), and 
information about prescribed medications (the prescription data and the classification of active ingredients of medications). As referral data were incompletely recorded, they were not extracted for this study. Patients' residential postcode was used to identify the corresponding Index of Relative Socioeconomic Disadvantage, a proxy for the socioeconomic status of each patient [19].

Descriptive analysis was used to describe the annual trends in the number of consultations, patients with MHCs, number of psychotropics prescribed by GPs, and annual trend in the number of psychotropic prescriptions across sub-categories - i.e. antidepressants, anxiolytics, antipsychotics, and antiepileptics. Negative binomial regression was used to explore changes of prescription patterns (annual psychotropic prescriptions per head of people with a MHC from GP clinics involved in MAGNET) over the study period, and to determine what patient characteristics were associated with the likelihood of receiving a prescription. The 0.05 level of statistical significance was adopted for each test. Results for the regression models were expressed as incidence rate ratios (IRR) with 95\% confidence interval (CIs). All analyses were conducted using Stata v13.0.

\section{Results}

From 2009 to 2014 there were 605,417 GP consultations for 77,466 patients aged 16-24 years with an average of 7.8 consultations per patient ( $95 \% \mathrm{CI}=7.73-7.91)$. Of 77,466 patients, $7892(10.1 \%)$ had been diagnosed with a MHC (63.9\% of these were female). Additionally, 1220 patients had been prescribed a psychotropic without a MHC diagnosis (60.9\% of these were female). Thus a total of $9112(11.8 \%)$ young patients were identified as potentially having a MHC in this study.

Among those 9112 young patients with MHC, consultations with female patients $(122,645)$ were higher than for male patients $(50,241)$. Female patients averaged 21.1 consultations (95\% CI $=20.4-21.7)$ during the period 2009-2014, compared to 14.8 consultations for male patients $(95 \% \mathrm{CI}=14.2-15.4)$. The majority of young patients who had at least two consultation records (78.9\%) visited only one medical clinic for their consultations. Table 2 presents these patient's demographic characteristics and mental health status. Nearly half of MHC patients (45.8\%) were non-smokers, $16.8 \%$ and $16.0 \%$ were ex-smokers and current smokers respectively (smoking status was not recorded for the remaining one fifth). Alcohol status was also not recorded for nearly $85 \%$ of the sample population; accordingly, alcohol and smoking status were not included in the analysis. Only $2.9 \%$ of patients were from areas with the lowest Index of Relative Socio-Economic Disadvantage quintile and about $88 \%$ were from the top two quintiles.
Table 2 Patient's demographic and mental health status

\begin{tabular}{lll}
\hline & \multicolumn{2}{l}{ Total $(\mathrm{N}=9112)$} \\
\cline { 2 - 3 } & $\mathrm{N}$ & $\%$ \\
\hline Gender (10missing) & & \\
Female & 5774 & 63.7 \\
Male & 3328 & 36.2 \\
Smoking status & & \\
Non-smoker & 4173 & 45.8 \\
Ex-smoker & 1531 & 16.8 \\
Current smoker & 1456 & 16.0 \\
Not recorded & 1952 & 21.4 \\
Alcohol status & & \\
Non-drinker & 358 & 3.9 \\
Drinker & 1054 & 11.6 \\
Not recorded & 7700 & 84.5 \\
Index of disadvantage quintilea(59 mssing) & & \\
1 & 261 & 2.9 \\
2 & 298 & 3.3 \\
3 & 560 & 6.2 \\
4 & 2580 & 29.0 \\
5 & 5311 & 58.7 \\
Mental health status & & \\
Diagnosed and prescribed psychotropic & 2747 & 30.1 \\
Diagnosed and not prescribed psychotropic & 5145 & 56.5 \\
Not diagnosed and prescribed psychotropic & 1220 & 13.4 \\
Mental health-related prescriptions & 11,934 & \\
arefer to Socio-Economic Indexes for Areas by postcode & \\
\hline
\end{tabular}

Over the study period 11,934 psychotropic prescriptions were initially provided to patients having a MHC; 95\% of these medication costs were subsidised (based on 2013-2014 prescription data). Among those 9112 patients with a MHC, 2747 patients (30.1\%) had a diagnostic label in the record and had been prescribed psychotropics, 5145 patients (56.5\%) had a diagnostic label in the record but had not been prescribed any psychotropic medication, and $13.4 \%$ of them had no diagnostic label in the record but had still been prescribed psychotropic medications (Table 2). During the study period, the number of consultations by patients with MHCs remained stable for both genders. As shown in Fig. 1, the number of patients with a MHC declined from 4394 in 2009 to 3816 in 2014. However, from 2009 to 2013 the number of psychotropics prescribed by GPs increased from 1791 to 2182 , before decreasing again to 2100 in 2014. Compared to the reference year 2009, the number of prescriptions per patient slightly increased each year (IRRs $1.04,95 \%$ CI: $0.93-1.15$ in 2010 to 1.35 , 95\% CI: $1.21-1.50$ in 2014) (Table 3). Women had a slightly higher rate of receipt of a prescription than men. 


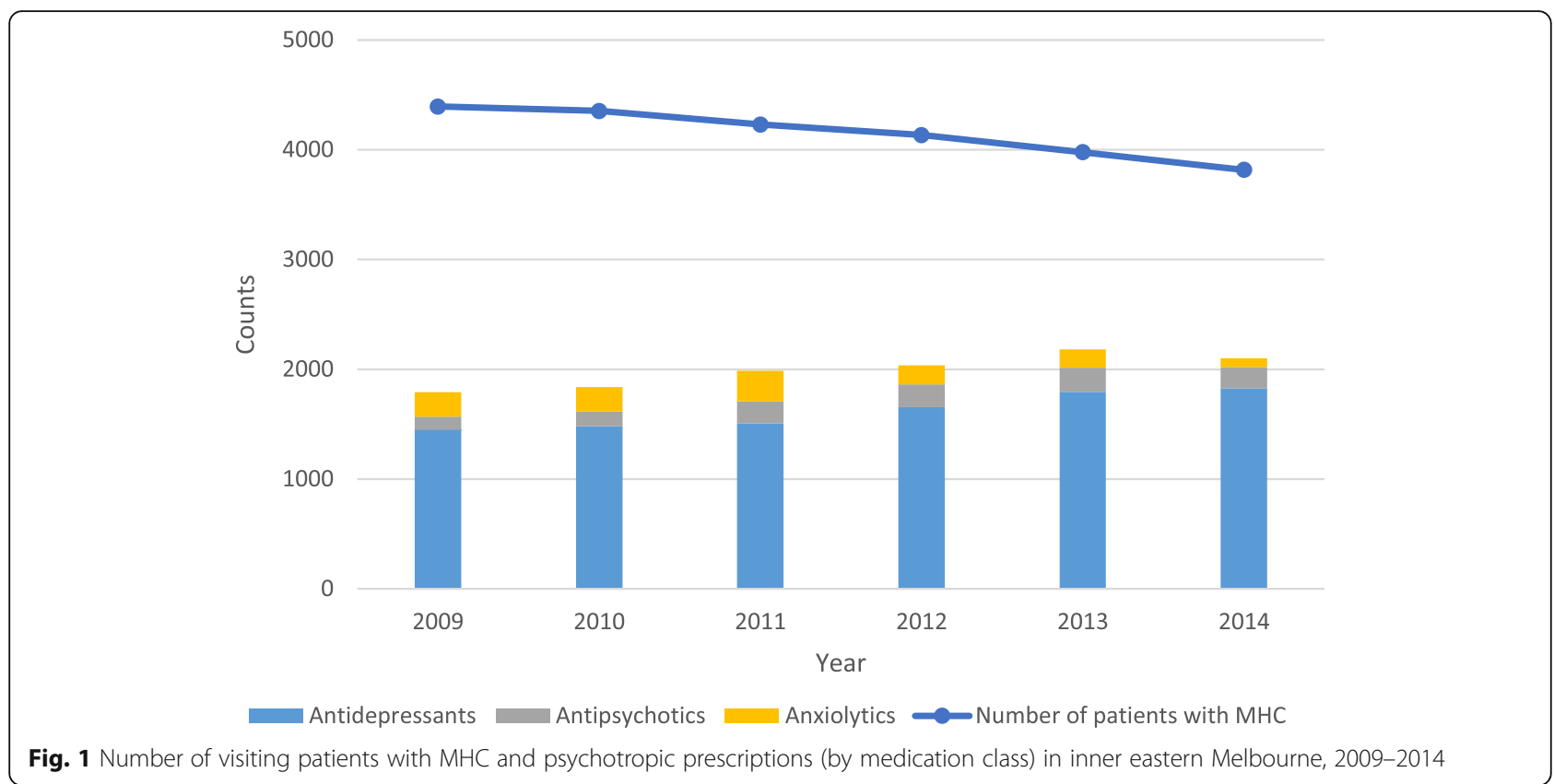

Patients aged $18-20$ and $21-24$ were 1.58 and 1.93 as likely respectively to be prescribed psychotropics when compared to those aged 16-17 (Table 3).

Antidepressants were the most commonly prescribed psychotropic (81.4\%), followed by anxiolytics $(9.6 \%)$ and antipsychotics (9.0\%) (Table 4). Antidepressant prescriptions increased by nearly $30 \%$ across all age groups from 2009 to 2014 (Fig. 1), contributing to the increase in overall prescriptions across the study period. Conversely, anxiolytics, which constituted $11.6 \%$ of total prescriptions in 2009 became the least commonly prescribed psychotropic in 2014, only constituting $3.6 \%$ of the total (Fig. 1).

SSRIs composed $75.1 \%$ of antidepressants prescribed and included escitalopram (37.2\%), sertraline (33.4\%), fluoxetine (17.0\%), citalopram (9.0\%) and fluvoxamine (3.3\%). Over $70 \%$ of the three most common SSRIs were prescribed to female patients and about $90 \%$ of them were prescribed to patients aged 18-24 years old at the visit. The most commonly prescribed SSRI to patients

Table 3 Amount, prescription rate per head of people with mental health condition, IRR of number of prescriptions in inner eastern Melbourne region, 2009-2014

\begin{tabular}{|c|c|c|c|c|c|}
\hline Category & Number of prescriptions & Number of patients & IRR & $95 \% \mathrm{Cl}$ & $p$-value \\
\hline \multicolumn{6}{|l|}{ Year of visit } \\
\hline 2009 & 1791 & 4394 & 1 (ref) & & \\
\hline 2010 & 1837 & 4353 & 1.04 & $0.93-1.15$ & 0.490 \\
\hline 2011 & 1987 & 4230 & 1.15 & $1.04-1.27$ & $0.009^{*}$ \\
\hline 2012 & 2037 & 4133 & 1.21 & $1.09-1.34$ & $<0.001^{*}$ \\
\hline 2013 & 2182 & 3977 & 1.35 & $1.21-1.49$ & $<0.001^{*}$ \\
\hline 2014 & 2100 & 3816 & 1.35 & $1.21-1.50$ & $<0.001^{*}$ \\
\hline \multicolumn{6}{|l|}{${ }^{\mathrm{a}}$ Gender } \\
\hline Male & 3850 & 3328 & 1 (ref) & & \\
\hline Female & 8078 & 5774 & 1.15 & $1.08-1.22$ & $<0.001^{*}$ \\
\hline \multicolumn{6}{|l|}{ Age group } \\
\hline $16-17$ years & 1074 & 2455 & 1 (ref) & & \\
\hline 18-20 years & 3442 & 4093 & 1.58 & $1.43-1.75$ & $<0.001^{*}$ \\
\hline $21-24$ years & 7418 & 6617 & 1.93 & $1.75-2.11$ & $<0.001^{*}$ \\
\hline
\end{tabular}

${ }^{\mathrm{a}} 10$ records with missing data were removed from analysis

${ }^{*} p<0.05$ level 
Table 4 Psychotropic prescriptions, by Anatomical Therapeutic Chemical (ATC) classification of medication prescribed in in inner eastern Melbourne region, 2009-2014

\begin{tabular}{|c|c|c|c|c|}
\hline Medication level 3 & Medication level 4 & $\mathrm{~N}$ & \% Subtotal & $\%$ Total \\
\hline \multirow[t]{5}{*}{ Antidepressants } & Selective Serotonin Reuptake Inhibitors (SSRIs) & 7288 & 75.1 & \multirow[t]{5}{*}{81.4} \\
\hline & Serotonin-norepinephrine reuptake inhibitors (SNRIs) & 1169 & 12.0 & \\
\hline & Other Antidepressants & 643 & 6.6 & \\
\hline & Tricyclic antidepressant. & 583 & 6 & \\
\hline & \multirow[t]{2}{*}{ Monoamine Oxidase Inhibitors } & 26 & \multirow[t]{2}{*}{0.3} & \\
\hline Subtotal & & 9709 & & \\
\hline Anxiolytics & \multirow[t]{2}{*}{ Benzodiazepine Derivatives } & 1147 & \multirow[t]{2}{*}{100} & \multirow[t]{2}{*}{9.0} \\
\hline Subtotal & & 1147 & & \\
\hline \multirow[t]{8}{*}{ Antipsychotics } & Diazepines, Oxazepines, Thiazepines and Oxepines. & 922 & 80.3 & \multirow[t]{8}{*}{9.6} \\
\hline & Lithium & 54 & & \\
\hline & Other Antipsychotics & 46 & 4.7 & \\
\hline & Benzamides & 29 & 2.5 & \\
\hline & Indole Derivatives & 12 & 1.0 & \\
\hline & Thioxanthene Derivatives & 7 & 0.6 & \\
\hline & Phenothiazines With Aliphatic Side-Chain & 5 & 0.4 & \\
\hline & Phenothiazines With Piperazine Structure & 3 & 0.2 & \\
\hline \multicolumn{2}{|l|}{ Subtotal } & \multicolumn{2}{|l|}{1078} & \\
\hline \multicolumn{2}{|l|}{ Total } & \multicolumn{2}{|l|}{11,934} & \\
\hline
\end{tabular}

16-17 years were sertraline (36.4\%), fluoxetine $(30.1 \%)$ and escitalopram (27.1\%).

SNRIs composed $12 \%$ of antidepressants prescriptions with venlafaxine being the only SNRI prescribed. About $72 \%$ of venlafaxine prescriptions were given to females, and $91 \%$ to patients aged $16-17$ years. Monoamine oxidase inhibitors were the least commonly prescribed antidepressants (Table 4). Benzodiazepine derivatives were the only anxiolytics prescribed by GPs. Diazepines and oxazepines (e.g. olanzapine and quetiapine) were the most common antipsychotics prescriptions prescribed and phenothiazine with aliphatic side-chain and phenothiazine with piperazine structure were the least common antipsychotics prescribed to young patients.

\section{Discussion}

Our study provides insights into recent trends in GP psychotropic prescribing for to young people aged 16-24 in the inner eastern region in Melbourne, Australia.

Our study suggests that approximately $12 \%$ of the 77,466 patients in the study catchment area had been identified by their GPs as having a mental health condition. This rate is slightly higher than national data, which reported that $9 \%$ of young people aged 1624 years had high or very high levels of psychological distress and $25 \%$ of them experienced at least one mental disorder [20]. However, as the MAGNET catchment area is socio-economically relatively prosperous, this difference may be on account of the overall higher rate of GP encounters with young people in areas of high socio-economic status compared to areas of lower socio-economic status rather than a true difference in prevalence rates [20]. Continuity of care was also high in this catchment area with this patient cohort. About $80 \%$ of patients visited only a single practice within the MAGNET group of practices in the inner eastern region of metropolitan Melbourne over the study period; overseas data indicates such relational continuity positively influences recovery from mental illness [21, 22].

Supporting previous evidence [23, 24] more females were diagnosed with a mental health condition than males, and females had greater numbers of consultations than males. This gender discrepancy could be because of a true difference in illness prevalence but it could also be an artifactual difference as female patients, compared to male patients, tend to report more mental illness symptoms (especially for depression) and present more often in general practice $[1,25,26]$. Thus, female patients' opportunities for diagnosis and prescription are increased [1, 25, 26]. GPs, in turn, may also be overresponding to young female patient's, and underresponding to young male patient's distress [26]. The evidence is equivocal in this area and qualitative analyses are needed to better understand the gendered dimensions of mental illness diagnosis and psychotropic prescribing for young patients in general practice. 
Patient's age also influenced GP prescribing with those aged 21-24 almost twice as likely to be prescribed a psychotropic as those aged under 16-17. This finding is consistent with previous research suggesting that GPs are reluctant to 'over-medicalise' young people [5]. Low rates of prescribing to this age group may also be influenced by the negative views held by young people about pharmaceutical treatments [6] and a preference for nonpharmaceutical intervention [7]. It may also relate to fears of inducing suicidality and aggression in young people prescribed SSRIs and SNRIs with recent evidence showing a doubling of these behaviours in young people using these forms of medication [27].

In conjunction, current guidelines [10-12] recommend non-pharmacological interventions as first-line treatments. Reflecting this, our data indicated that over half of all diagnosed patients were not prescribed any psychotropic medication at all. Also concordant with clinical guidelines [10] was a marked reduction in prescription of anxiolytics or benzodiazepines over the study period, likely reflecting increased GP awareness of the potential harms of these medications and risk of abuse especially in young patients.

It is also possible that the Better Access scheme, which provides Government subsidised access to up to 10 individual visits to specialist mental health services per calendar year, may have influenced low rates of GP prescribing. However, qualitative research with GPs is needed to verify whether this is true or not. Other Australian studies $[16,17]$ examining adult populations show that psychotropic prescribing increased following the introduction of the Better Access scheme, especially for antidepressants. Likewise, in our study, among those receiving GP prescriptions, the psychotropic prescribing rate increased by $32 \%$ over six years across all age groups, mainly for antidepressants. Australian [28], UK [29], and US [30] studies note increased reliance on antidepressants for treating mental disorders despite concerns of low therapeutic benefit for mild-moderate depression and the potential for adverse reactions such as increased suicidality in young people [27, 31, 32]. SSRI prescriptions increased the most within the antidepressant category, specifically escitalopram, sertraline, and fluoxetine. Current clinical guidelines [11, 14] only recommend use of fluoxetine in adolescents because neither escitalopram nor sertraline have garnered sufficient evidence on which to base recommendations. In our study the rate of fluoxetine prescriptions to patients aged 16-17 years was higher (30.1\%) than reported in Karanges et al.'s [1] analyses of 2009-2012 prescribing trends (19.4\%), which could suggest that GP prescribing may be aligning with guideline recommendations over time. On the other hand, the overall rate of fluoxetine prescribing in our study was only $17 \%$, superseded by escitalopram and sertraline. Reasons for the high prescription rates of these two medications in patients under 18 years is unclear; also unclear is why venlafaxine was the only SNRI to be prescribed to young patients. While venlafaxine has the highest dispensation rate of all SNRIs and is the third most commonly dispensed antidepressant in Australia, it, like other SNRIs, has been reported to cause more complex side-effects than SSRIs [17].

Our study has limitations. As mentioned, the data from MAGNET is restricted to inner eastern Melbourne region, an economically prosperous area where most patients have a high socio-economic status. This regional specificity restricts our capacity to comment on associations between low socio-economic status, MHCs, and GP prescribing. Moreover, patients may visit other practices outside the region during the study period, which limits the generalisability of the findings. Referral data were also incompletely recorded, limiting our ability to explore non-pharmacological interventions GPs utilised in their management of youth mental health. Despite a strong association between substance use and mental illness especially among young people [33], GP records were sparse with regards to their young patient's smoking and alcohol use; such an absence could indicate that GPs fail to ask about these risk factors or that patients are reluctant to disclose their use or other mitigating factors. In any case, poor GP data records limit our ability to make inferences around mental illness, psychotropic prescriptions, and substance use. Our analysis also did not take into account repeat prescription since detailed information was not provided. Patients having MHCs were identified by coding relevant key words from GPs' 'free text' manual entries in the diagnosis category of the patient's record, and thus the quality of coding and recording were unknown. This might explain why 1220 patients were prescribed psychotropics but not diagnosed with a MHC. Coding ambiguities around diagnostic categories also limited our ability to investigate how prescribed medications directly related to diagnosed MHCs - i.e. appropriate prescribing. Another limitation should be noted is that GP might prescribe psychotropics for other health conditions (off-label prescribing), which also affect our assumption of identifying MHC patients.

\section{Conclusion}

The degree of accord between our findings and the published literature underwrites the validity and reliability of our findings, and also provides a timely update in the scholarship around GP psychotropic prescribing for youth mental health. Our findings show that overall, GP prescribing of psychotropics for young people reflected guideline recommendations. However, there were some 
discrepancies between GP's antidepressant prescribing and guideline recommendations, reasons for which were unclear. Research is needed to investigate GPs decisionmaking processes underlying their prescribing, to target interventions to improve existing data in GP records to improve management, and to identify areas of further training if needed to facilitate greater concordance between clinical practice and guideline recommendations. Such training might include not only more appropriate prescribing for particular age groups but also training on how to initiate conversations around de-prescribing and safely discontinuing medicines. Arguably, the latter intervention is of greater importance given the growing global reliance on antidepressants for treating common mental disorders despite limited therapeutic benefit for mild-moderate depression and its iatrogenic effects in young people [31,32]. While the evidence is limited on how precisely to implement antidepressant discontinuation [34], unequivocally GPs have a strong role to play in the process as facilitators of safe discontinuation and managing discontinuation symptoms [35, 36]. Given that GPs are one of the most frequently utilised health care providers amongst young people [20,37] and that the vast majority of young people are not in receipt of specialist mental health care, visits to their GP represents an important opportunity for intervention. Accordingly, GPs must be supported to continuously improve their patient care.

\section{Abbreviations}

Cl: Confidence interval; GP: General practitioner; IRR: Incidence rate ratios; MAGNET: Melbourne East Monash general practice dataset; MHC: Mental health condition; SNRI: Serotonin-norepinephrine reuptake inhibitors; SSRI: Selective serotonin reuptake inhibitors

\section{Acknowledgements}

The authors acknowledge the Melbourne East Monash General Practice Database and MAGNET Scientific Committee for facilitating access to the data.

\section{Funding}

This study was funded by Faculty Strategic Grant Scheme, Monash University (SGS150116)

\section{Availability of data and materials}

All authors had full access to, statistical reports and tables in the study. The data that support the findings of this study are available from the Melbourne East Monash General Practice Database but restrictions apply to the availability of these data, which were used under license for the current study, and so are not publicly available. Data are however available from the authors upon reasonable request and with permission of the Melbourne East Monash General Practice Database and MAGNET Scientific Committee.

\section{Authors' contributions}

BB devised the study, led the analysis and writing, and organised submission. TX undertook data analysis and wrote the initial draft of the article methods and findings. LT extracted the data and contributed to data analysis and interpretation. DM contributed to data analysis and clinical interpretation of results. All authors read and approved the final manuscript.

\section{Competing interests}

DM is the scientific director of MAGNET. LT is the chief statistician for MAGNET.

\section{Consent for publication}

Not applicable.

\section{Ethics approval and consent to participate}

This study received ethic approval by the Monash University Human Research Ethic Committee, Australia (CF15/998-2015000463).

\section{Publisher's Note}

Springer Nature remains neutral with regard to jurisdictional claims in published maps and institutional affiliations.

Received: 17 October 2016 Accepted: 31 May 2017

Published online: 06 June 2017

\section{References}

1. Karanges EA, Stephenson CP, McGregor IS. Longitudinal trends in the dispensing of psychotropic medications in Australia from 2009-2012: focus on children, adolescents and prescriber specialty. Aust N Z J Psychiatry. 2014;48(10):917-31.

2. Roberts $\mathrm{JH}$, Crosland A, Fulton J. Patterns of engagement between GPs and adolescents presenting with psychological difficulties: a qualitative study. Br J Gen Pract. 2014;64(622):e246-54.

3. Glozier N, Davenport T, Hickie IB. Identification and management of depression in Australian primary care and access to specialist mental health care. Psychiatr Serv (Washington, DC). 2012;63(12):1247-51.

4. Fleury M-J, Farand L, Aube D, Imboua A. Management of mental health problems by general practitioners in Quebec. Can Fam Physician. 2012; 58(12):e732-8.

5. Vallance AK, Kramer T, Churchill D, Garralda ME. Managing child and adolescent mental health problems in primary care: taking the leap from knowledge to practice. Prim Health Care Res Dev. 2011;12(4):301-9.

6. Wright A, Harris MG, Wiggers JH, Jorm AF, Cotton SM, Harrigan SM, et al. Recognition of depression and psychosis by young Australians and their beliefs about treatment. Med J Aust. 2005;183(1):18-23.

7. Burns J, Birrell E. Enhancing early engagement with mental health services by young people. Psychol Res Behav Manag. 2014;7:303-12.

8. The MaGPle Research Group. The treatment of common mental health problems in general practice. Fam Pract. 2006;23(1):53-9.

9. Gibbons RD, Brown CH, Hur K, Marcus SM, Bhaumik DK, Erkens JA, et al. Early evidence on the effects of regulators' Suicidality warnings on SSRI prescriptions and suicide in children and adolescents. Am J Psychiatr. 2007; 164(9):1356-63.

10. The Royal Australian College of General Practitioners. Clinical practice guidelines: Prescribing drugs of dependence in general practice, Part B Benzodiazepines. Melbourne: 2015.

11. beyondblue: The national depression initiative. Clinical practice guidelines: Depression in adolescents and young adults. Melbourne: 2010.

12. Committee for Therapeutic Interventions and Evidence Based Practice and Faculty of Child and Adolescent Psychiatry. Professional practice guideline 7: guidance for psychotropic medication use in children and adolescents. Melbourne: The Royal Australian and New Zealand College of Psychiatrists; 2015.

13. American Academy of Child and Adolescent Psychiatry. Practice parameter for the use of atypical antipsychotic medications in children and adolescents. Washington DC: 2011

14. Brown G, Atkinson J, Jenkins P, Hewitt J. Guidance on the use of antidepressants in children and adolescents. London: NHS Foundation Trust; 2014

15. Hickie IB, Fogarty AS, Davenport TA, Luscombe GM, Burns J. Responding to experiences of young people with common mental health problems attending Australian general practice. Med J Aust. 2007;187(7):S47-52.

16. Britt H, Miller GC, Henderson J, Bayram C, Harrison C, Valenti L, et al. General practice activity in Australia: 2013-14. Sydney: University of Sydney press; 2014

17. Stephenson CP, Karanges E, McGregor IS. Trends in the utilisation of psychotropic medications in Australia from 2000 to 2011. Aust N Z J Psychiatry. 2013;47(1):74-87.

18. Health Language Analytics Global: Common medical Classification. In.; 2016. 
19. Census of Population and Housing: Socio-Economic Indexes for Areas (SEIFA), Australia, 2011

20. Australian Institute of Health and Welfare. Young Australians: their health and wellbeing 2011. Canberra: AlHW; 2011.

21. Green CA, Polen MR, Janoff SL, Castleton DK, Wisdom JP, Vuckovic N, et al. Understanding how clinician-patient relationships and relational continuity of care affect recovery from serious mental illness: STARS study results. Psychiatr Rehabil J. 2008;32(1):9-22.

22. Nadelson C, Notman MT. Boundaries in the doctor-patient relationship. Theor Med Bioeth. 2002;23(3):191-201.

23. Patton GC, Coffey C, Romaniuk H, Mackinnon A, Carlin JB, Degenhardt L, et al. The prognosis of common mental disorders in adolescents: a 14-year prospective cohort study. Lancet. 2014;383(9926):1404-11.

24. Landstedt E, Coffey J, Nygren M. Mental health in young Australians: a longitudinal study. J Youth Stud. 2015:1-13.

25. Hohmann AA. Gender bias in psychotropic drug prescribing in primary care. Med Care. 1989;27(5):478-90.

26. Hooper LM. The unmet needs of depressed adolescent patients: how race, gender, and age relate to evidence-based depression care in rural areas. Prim Health Care Res Dev. 2010;11(4):339-48.

27. Sharma T, Guski LS, Freund N, Gotzsche PC. Suicidality and aggression during antidepressant treatment: systematic review and meta-analyses based on clinical study reports. BMJ. 2016;352:165.

28. Hollingworth SA, Burgess PM, Whiteford HA. Affective and anxiety disorders: prevalence, treatment and antidepressant medication use. Aust N Z J Psychiatry. 2010;44(6):513-9.

29. Ilyas S, Moncrieff J. Trends in prescriptions and costs of drugs for mental disorders in England, 1998-2010. Br J Psychiatry. 2012;200(5):393-8.

30. Olfson M, Marcus SC. National patterns in antidepressant medication treatment. Arch Gen Psychiatry. 2009;66(8):848-56.

31. Karanges E, McGregor I. Antidepressants and adolescent brain development. Future Neurol. 2011;6:783-808.

32. Fournier JC, DeRubeis RJ, Hollon SD, Dimidjian S, Amsterdam JD, Shelton $\mathrm{RC}$, et al. Antidepressant drug effects and depression severity: a patient-level meta-analysis. JAMA. 2010;303(1):47-53.

33. Reavley NJ, Cvetkovski S, Jorm AF, Lubman DI. Help-seeking for substance use, anxiety and affective disorders among young people: results from the 2007 Australian National Survey of mental health and wellbeing. Aust N Z J Psychiatry. 2010;44(8):729-35.

34. Wilson $\mathrm{E}$, Lader M. A review of the management of antidepressant discontinuation symptoms. Ther Adv Psychopharmacol. 2015;5(6):357-68.

35. Verbeek-Heida PM, Mathot EF. Better safe than sorry-why patients prefer to stop using selective serotonin reuptake inhibitor (SSRI) antidepressants but are afraid to do so: results of a qualitative study. Chronic IIIn. 2006;2(2):133-42.

36. Leydon GM, Rodgers L, Kendrick T. A qualitative study of patient views on discontinuing long-term selective serotonin reuptake inhibitors. Fam Pract. 2007;24(6):570-5

37. Van Dyke N, Maddern CM, Walker R, Reibel T. Young People's experiences with health services - final report. Commissioner for Children and Young People. Perth, WA: 2014.

\section{Submit your next manuscript to BioMed Central and we will help you at every step:}

- We accept pre-submission inquiries

- Our selector tool helps you to find the most relevant journal

- We provide round the clock customer support

- Convenient online submission

- Thorough peer review

- Inclusion in PubMed and all major indexing services

- Maximum visibility for your research

Submit your manuscript at www.biomedcentral.com/submit
Biomed Central 